\title{
OPEN Deep learning-based model for detecting 2019 novel coronavirus pneumonia on high-resolution computed tomography
}

Jun Chen ${ }^{3,9}$, Lianlian Wu U,6,7,9 Jun Zhang $^{1,6,7,9}$, Liang Zhang ${ }^{3}$, Dexin Gong ${ }^{1,6,7}$, Yilin Zhao ${ }^{3}$, Oiuxiang Chen ${ }^{4}$, Shulan Huang ${ }^{4}$, Ming Yang ${ }^{4}$, Xiao Yang ${ }^{4}$, Shan $\mathrm{Hu}^{5}$, Yonggui Wang ${ }^{8}$, Xiao Hu ${ }^{5}$, Biqing Zheng ${ }^{5}$, Kuo Zhang ${ }^{5}$, Huiling Wu ${ }^{1,6,7}$, Zehua Dong ${ }^{1,6,7}$, Youming Xu ${ }^{1,6,7}$, Yijie Zhu ${ }^{1,6,7}$, Xi Chen ${ }^{1,6,7}$, Mengjiao Zhang ${ }^{1}$, LileiYu ${ }^{1 凶}$, Fan Cheng ${ }^{2 \bowtie}$ \& Honggang Yu ${ }^{1,6,7 凶}$

Computed tomography (CT) is the preferred imaging method for diagnosing 2019 novel coronavirus (COVID19) pneumonia. We aimed to construct a system based on deep learning for detecting COVID19 pneumonia on high resolution CT. For model development and validation, 46,096 anonymous images from 106 admitted patients, including 51 patients of laboratory confirmed COVID-19 pneumonia and 55 control patients of other diseases in Renmin Hospital of Wuhan University were retrospectively collected. Twenty-seven prospective consecutive patients in Renmin Hospital of Wuhan University were collected to evaluate the efficiency of radiologists against 2019-CoV pneumonia with that of the model. An external test was conducted in Qianjiang Central Hospital to estimate the system's robustness. The model achieved a per-patient accuracy of $95.24 \%$ and a per-image accuracy of $98.85 \%$ in internal retrospective dataset. For 27 internal prospective patients, the system achieved a comparable performance to that of expert radiologist. In external dataset, it achieved an accuracy of $96 \%$. With the assistance of the model, the reading time of radiologists was greatly decreased by $65 \%$. The deep learning model showed a comparable performance with expert radiologist, and greatly improved the efficiency of radiologists in clinical practice.

In December 2019, a new coronavirus infection disease (hereinafter referred to as COVID-19) was first reported in Wuhan. Subsequently, the outbreak began to spread widely in China and even abroad ${ }^{1-3}$.

The clinical manifestations of the COVID-19 pneumonia is complicated and could be characterized as fever, cough, myalgia, headache, and gastrointestinal symptoms onset ${ }^{4}$. Although the nucleic acid detection was considered determinant for identifying the COVID-19 infection and more rapid detection kit for the novel coronavirus has come into mass production, computed tomography (CT) scan is still the most efficient modality for detecting and evaluating the severity of pneumonia ${ }^{5}$. An update series demonstrate that CT findings were positive in all 140 laboratory-confirmed COVID-19 patients, even in the early stage ${ }^{4,6}$. In the fifth version of diagnostic manual of COVID-19 launched by the National Health and Health Commission of China, the radiographic characteristics of pneumonia was included the clinical diagnostic standard in Hubei Province ${ }^{7}$. Subsequently, 14,840 new cases

\footnotetext{
${ }^{1}$ Department of Internal Medicine, Renmin Hospital of Wuhan University, 99 Zhangzhidong Road, Wuhan 430060, Hubei Province, China. ${ }^{2}$ Department of Urinary Surgery, Renmin Hospital of Wuhan University, 99 Zhangzhidong Road, Wuhan 430060, Hubei Province, China. ${ }^{3}$ Department of Radiology, Renmin Hospital of Wuhan University, Wuhan, China. ${ }^{4}$ Qianjiang Central Hospital, Qianjiang, China. ${ }^{5}$ Wuhan EndoAngel Medical Technology Company, Wuhan, China. ${ }^{6}$ Hubei Provincial Clinical Research Center for Digestive Disease Minimally Invasive Incision, Renmin Hospital of Wuhan University, Wuhan, China. ${ }^{7}$ Key Laboratory of Hubei Province for Digestive System Disease, Renmin Hospital of Wuhan University, Wuhan, China. ${ }^{8}$ Hubei Key Laboratory of Critical Zone Evolution, School of Geography and Information Engineering, China University of Geosciences, Wuhan, China. ${ }^{9}$ These authors contributed equally: Jun Chen, Lianlian Wu and Jun Zhang. ${ }^{\varpi}$ email: lileiyu@whu.edu.cn; urology1969@ aliyun.com; yuhonggang1968@163.com
} 
of COVID-19 were reported within 1 day on Feb 13, 2020 in Wuhan, including 13,332 cases of clinical diagnoses ${ }^{8}$. This highlighted the importance of CT in the diagnosis of COVID-19 pneumonia.

Due to the outbreak of the COVID-19, thousands of patients waited in line for CT examination in the designated fever outpatient hospital at Wuhan and other cities. As of Feb 14, there are 5,534 suspected cases, 38,107 confirmed patients receiving treatment in hospital, and 77,323 cases under medical observation in Hubei province ${ }^{9}$. Most of them need to undergo CT examination, however, there are less than 4,500 radiologists in cities of Hubei according to the China Health Statistical Yearbook $(2018)^{10}$. Meanwhile, because the lung infection foci are small in the early stage of the COVID-19 infection, thinner layer ( $2.5 \mathrm{~mm}, 1.25 \mathrm{~mm}$ or even $0.625 \mathrm{~mm})$ scanning were usually needed instead of conventional CT scan $(5 \mathrm{~mm})$ for diagnosis, which would be more timeconsuming. All these made radiologists overloaded, delay the diagnosis and isolation of patients, affect patient's treatment and prognosis, and ultimately, affect the control of COVID-19 epidemic.

Deep learning, an important breakthrough in the domain of AI in the past decade, has huge potential at extracting tiny features by the basic unit of DCNN's sampling kernel in image analysis ${ }^{11}$. Our group also succeeded in recruiting this technique in minor lesion detection and real-time assistance to doctors in gastrointestinal endoscopy ${ }^{12-16}$.

In the present research, we construct and validate a system based on deep learning for identification of viral pneumonia on CT. Our model has comparable performance with expert radiologist, but take much less time. The module and source code developed in this work were shared for global researches in https:/github.com/ endo-angel/ct-angel, and an open-access website has been made available to provide free provide to the present system (https://121.40.75.149/znyx-ncov/index).

\section{Method}

Patients. We first retrospectively collected 46,096 anonymous images from 106 admitted patients, including 51 patients of laboratory confirmed COVID-19 pneumonia and 55 control patients of other diseases in Renmin Hospital of Wuhan University (Wuhan, Hubei province, China) for model development. The patients' CT scan images and reports, history, clinical manifestations, physical findings, and viral pathogen results were all collected. For prospective patients, 27 consecutive patients undergoing CT scans were enrolled in the designated CT rooms in Feb 5, 2020 in Renmin Hospital of Wuhan University.

This study was approved by the Ethics Committee of both Renmin Hospital of Wuhan University and Qianjiang Central Hospital. Written informed consent was provided by all prospective participants. Because of virus contamination, the signed informed consents were carefully sealed and kept in the specific place according to the Law of the People's Republic of China on Infectious Disease Prevention and Control ${ }^{17}$. For patients whose CT scans were stored in the retrospective databases, informed consent was waived by the Ethics Committee. A statement to confirm that all methods were carried out in accordance with relevant guidelines and regulations.

Diagnostic testing for COVID-19. Patient's respiratory secretions were collected and transferred to a sterile test tube with a virus transport medium. Fluorescent RT-PCR analysis of samples was performed using the COVID-19 nucleic acid detection kit developed by Shanghai Geneodx Biotechnology Co., Ltd. This detection kit was approved by the US National Drug Administration (NMPA) on January 26, 2019 and recommended by the Centers for Disease Control and Prevention (CDC) ${ }^{18}$. The rapid, high-precision COVID-19 detection kit greatly accelerated the confirmation of human COVID-19 infection.

Datasets. As shown in Fig. 1, a total of 46,096 CT scan images from 51 COVID-19 pneumonia patients and 55 control patients of other disease from Renmin Hospital of Wuhan University were collected for developing the model to detect COVID-19 pneumonia. After filtering those images without good lung fields, 35,355 images were selected and split into training and retrospectively testing datasets. Enrolled images in training dataset covered almost all common CT features of COVID19 pneumonia, as presented in Fig. 2. Three radiologists with more than 5 years of clinical experience labelled infection lesions of COVID-19 pneumonia patients in training dataset, and selected images containing COVID19 pneumonia lesions in testing set, and their labels were combined by consensus. For prospectively testing the model, 13,911 images of 27 consecutive patients undergoing CT scans in Feb 5, 2020 in Renmin Hospital of Wuhan University were further collected. All CT scans were obtained in Renmin Hospital of Wuhan University. To estimate the robustness of the system, an external dataset containing 100 patients (13,734 images from 50 COVID-19 and 17,030 images from 50 normal control patients) were retrospectively collected from Qianjiang Central Hospital, China. The instruments used in this study included Optima CT680, Revolution CT and Bright Speed CT scanner (all GE Healthcare).

Training algorithm. This work is built on the top of UNet++, a novel and powerful architecture for medical image segmentation ${ }^{19}$, for the identification. Resnet-50 was used as backbone of UNet++ as previously described ${ }^{20}$. ResNet- $50^{21}$ was pretrained using ImageNet dataset ${ }^{22}$, and all the pre-training parameters of ResNet-50 are loaded to UNet++. The network architecture of UNet++ was shown in Fig. 3. Briefly, UNet++ consists of encoder and decoder connecting through a series of nested dense convolutional blocks. The semantic gap between the feature maps of the encoder and decoder is bridged prior to fusion. The encoder extract features by down-sampling; the decoder map features to the original image by up-sampling, make classification by pixels, and thus achieve the purpose of segmentation. We first trained UNet++ to extract valid areas in CT images using 289 randomly selected CT images and tested it in other 600 randomly selected CT images. The training images were labelled with the smallest rectangle containing all valid areas by researchers. The model successfully extracted valid areas in 600 images in testing set with an accuracy of $100 \%$. For detecting suspicious lesions on CT scans, 691 images of COVID-19 pneumonia infection lesions labelled by radiologists and 300 images ran- 


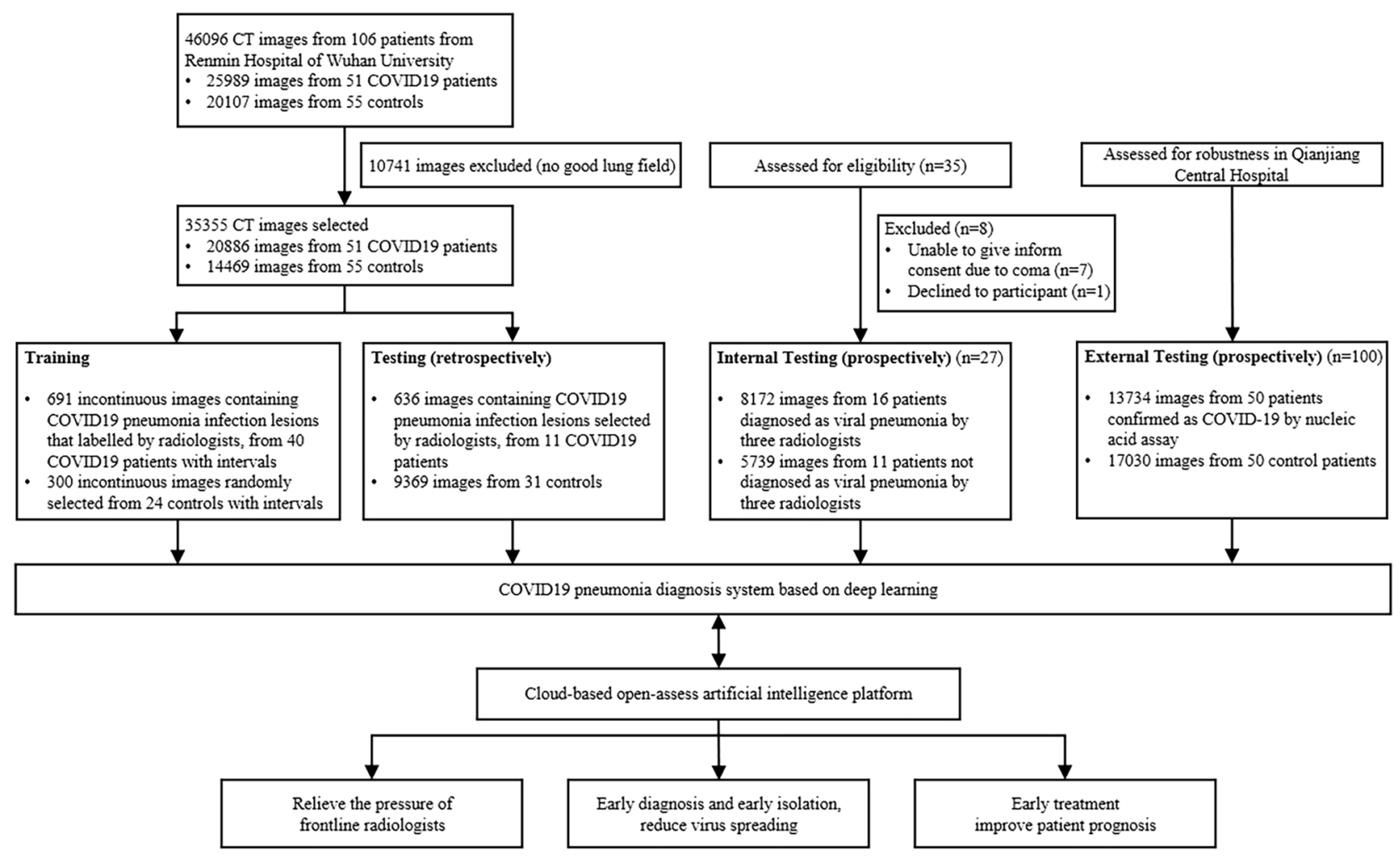

Figure 1. Workflow diagram for the development and evaluation of the model for detecting COVID19 pneumonia.

domly selected from patients of non-COVID-19 pneumonia were used. Taking the raw CT scan images as input with a resolution of $512 \times 512$, and the labelled map from the expert as output, UNet++ was used to train in Keras in an image-to-image manner. The suspicious region was predicted under a confidence cutoff value of 0.50 , and a prediction box pixel of over 25 . The training curves of UNet++ for extracting valid areas and detecting suspicious lesions in CT images were shown in Supplementary Figure 1 and Supplementary Figure 2, respectively. The prediction schematic of the model was shown in Fig. 4. Raw images were firstly input into the model, and after processing of the model, prediction boxes framing suspicious lesions were output. Valid areas were further extracted and unnecessary fields were filter out to avoid possible false positives. To predict by case, a logic linking the prediction results of consecutive images was added. CT images with the above prediction results were divided into four quadrants, and results would be output only when three consecutive images were predicted to have lesions in the same quadrant.

Testing of the model in retrospective data. To evaluate the performance of the model on CT scan images, five metrics including the accuracy, sensitivity, specificity, positive prediction value (PPV) and negative prediction value (NPV) were calculated as follows: accuracy = true predictions/total number of cases, sensitivity $=$ true positive/positive, specificity $=$ true negative/negative, $\mathrm{PPV}=$ true positive $/$ (true positive + false positive), NPV = true negative/(true negative + false negative). The "true positive" is the number of correctly predicted COVID-19 pneumonia cases/images, "false positive" is the number of mistakenly predicted COVID-19 pneumonia cases/images, "positive" is the number of cases/images of COVID-19 pneumonia patients, "true negative" is the number of correctly predicted non-COVID-19 pneumonia cases/images, "false negative" is the number of mistakenly predicted non-COVID-19 pneumonia cases/images and 'negative' is the number of nonCOVID-19 pneumonia cases/images enrolled. For image-based metrics, 636 images containing infection lesions identified by radiologists among 11 patients of COVID-19 pneumonia were used as the positive sample, and 9369 CT scan images from 31 patients of non-COVID-19 pneumonia were used as the negative sample.

Evaluating the efficiency of radiologist in the traditional way. To evaluate the performance and cost of time of radiologist against 2019-CoV pneumonia, prospectively consecutive patients undergoing CT scans were enrolled in the designated CT rooms in Feb 5, 2020 in Renmin Hospital of Wuhan University. An expert radiologist was required to read all CT images of enrolled patients using the working computer, and determine if each patient has viral pneumonia. The research assistant used a stopwatch to record the expert's reading time. The expert radiologist was associate chief physician of the Radiology Department of Renmin Hospital of Wuhan University, with clinical experience of 30 years, and independently diagnosed about 300 viral pneumonia. Hospitalized viral pneumonia cases judged by radiologists were all diagnosed using COVID-19 

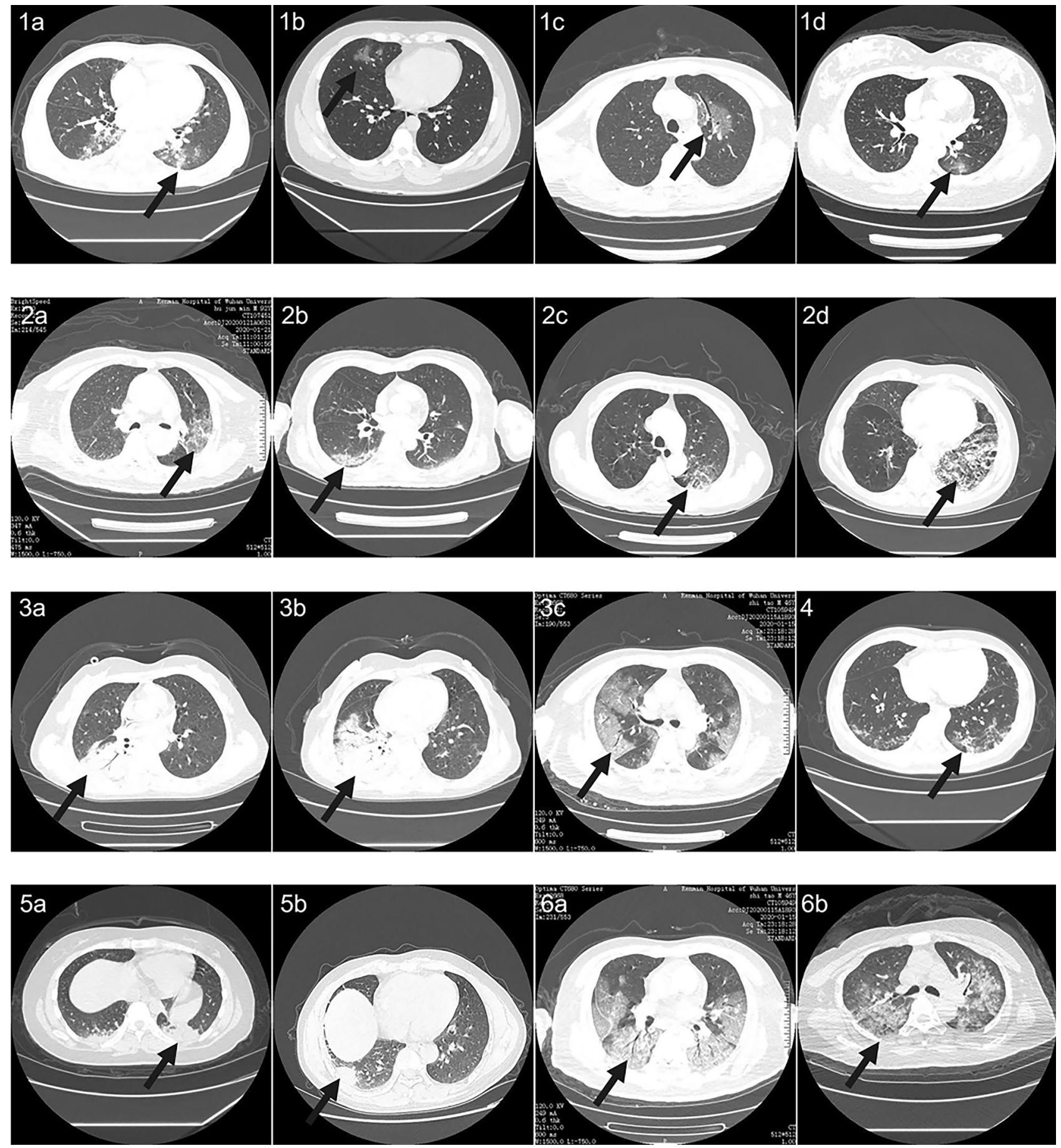

Figure 2. Representative images of COVID19 pneumonia. More than six common Computed tomography (CT) features of COVID19 pneumonia were covered in selected images. 1(a-d), the lesions were mainly ground-glass-like, with thickened blood vessels walking and including gas-bronchial signs in 1(c); 2(a-d), the lesions were mainly ground glass changes, and paving stone-like changes were observed on $2(\mathbf{d}) ; 3(\mathbf{a}-\mathbf{c})$, the lesions become solid with a large range, and air-bronchial signs are seen inside; 4 , the lesion is located in the lower lobe of both lungs, and is mainly grid-like change with ground glass lesion; $5(\mathbf{a}, \mathbf{b})$, the lesions are mainly consolidation; $6(\mathbf{a}, \mathbf{b})$, the lesions are mainly large ground glass shadows, showing white lung-like changes, with air-bronchial signs. 


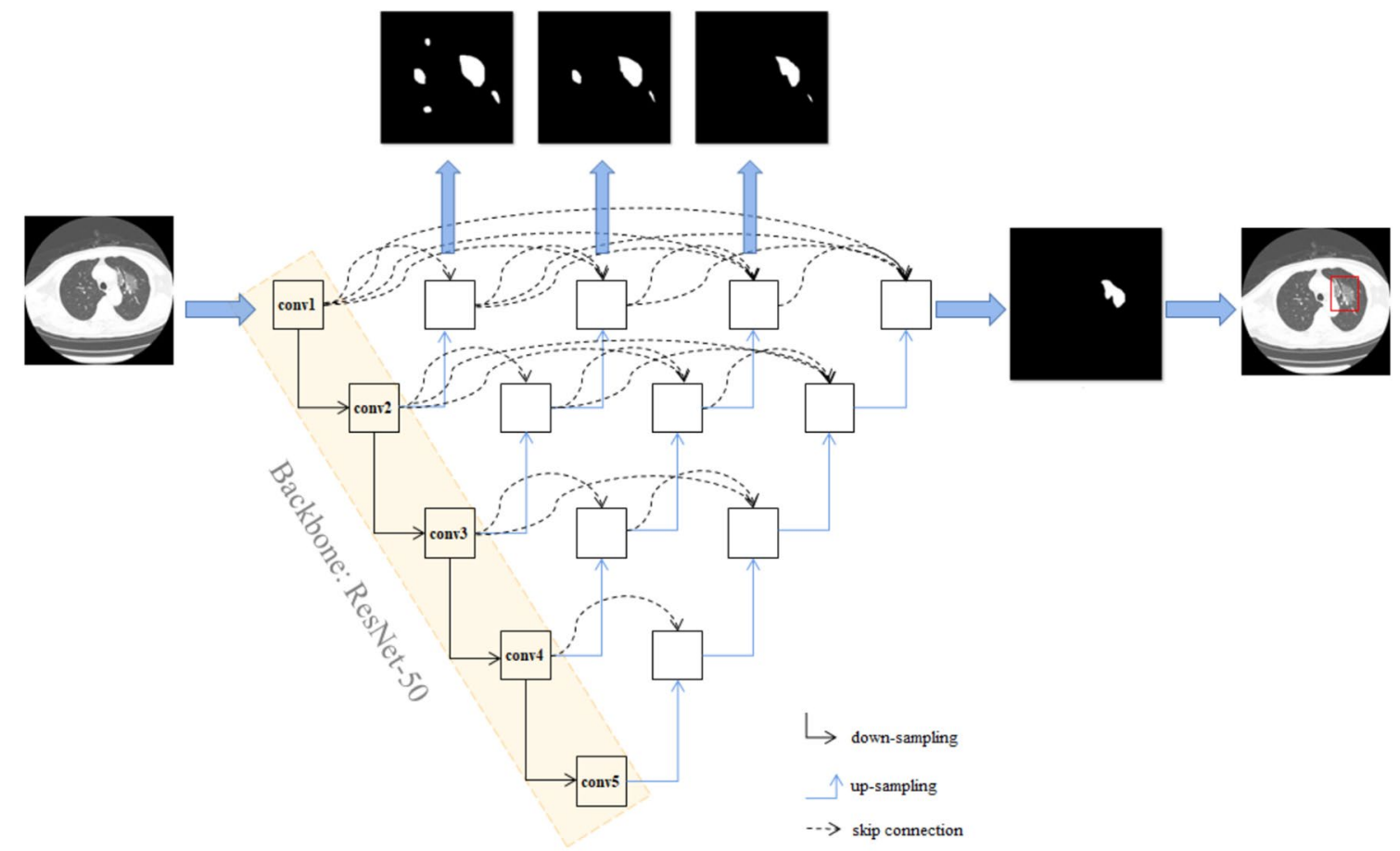

Figure 3. The network architecture of UNet++. UNet++ consists of encoder and decoder connecting through a series of nested dense convolutional blocks. The semantic gap between the feature maps of the encoder and decoder is bridged prior to fusion. The encoder extract features by down-sampling; the decoder map features to the original image by up-sampling, make classification by pixels, and thus achieve the purpose of segmentation. Resnet-50 was used as backbone of UNet++, and all the pre-training parameters of ResNet-50 are loaded to UNet++.

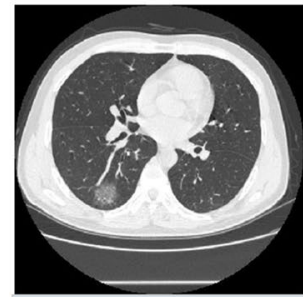

Input CT images

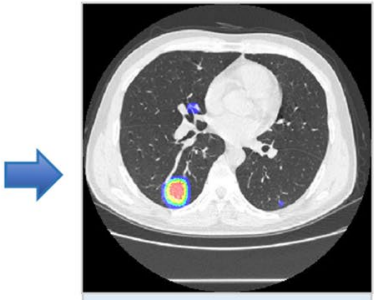

Activation map

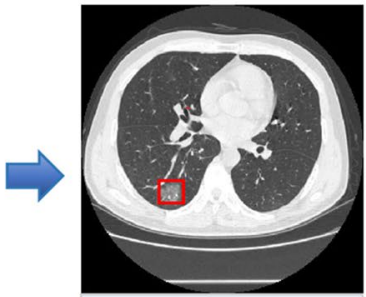

Prediction box

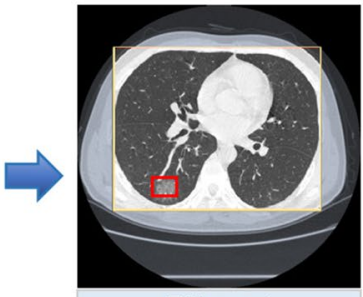

Filter out unnecessary fileds

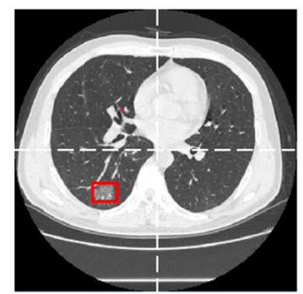

Four quadrant division
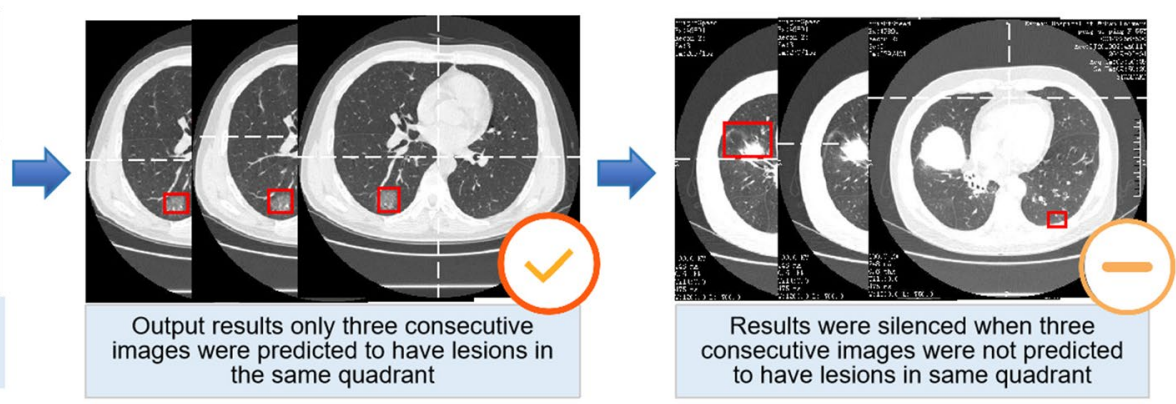

Figure 4. Processing and prediction schematic of the model. Raw images were firstly input into the model, and after processing of the model, prediction boxes framing suspicious lesions were output. Valid areas were further extracted and unnecessary fields were filter out to avoid possible false positives. To predict by case, a logic linking the prediction results of consecutive images was added. Computed tomography (CT) images with the above prediction results were divided into four quadrants, and results would be output only when three consecutive images were predicted to have lesions in the same quadrant. 


\begin{tabular}{|l|l|l|l|}
\hline \multicolumn{5}{|l|}{} & All patients $(\mathbf{n}=51)$ & Training set (n=40) & Testing set (n=11) \\
\hline Age, years, median (IQR) & $52(38,69)$ & $54.5(41.5,71.25)$ & $42(34.5,65.5)$ \\
\hline Sex, n (\%) & $18(35.3)$ & $11(27.5)$ & $7(63.6)$ \\
\hline Men & $33(64.7)$ & $29(72.5)$ & $4(36.4)$ \\
\hline Women & $28(54.9)$ & $21(52.5)$ & $7(63.6)$ \\
\hline Presenting symptoms and signs onset, n (\%) & $27(52.9)$ & $20(50)$ & $7(63.6)$ \\
\hline Fever & $7(13.7)$ & $6(15)$ & $1(9.1)$ \\
\hline Cough & $6(11.8)$ & $6(15)$ & 0 \\
\hline Chest tightness or pain & $10(19.6)$ & $8(20)$ & $2(18.2)$ \\
\hline Dyspnea & $12(23.5)$ & $10(25)$ & $2(18.2)$ \\
\hline Muscle soreness & $3(5.9)$ & $2(5)$ & $1(9.1)$ \\
\hline Expectoration & $6(11.8)$ & $6(15)$ & 0 \\
\hline Headache & \multicolumn{3}{l}{} \\
\hline Digestive symptoms & $18(35.3)$ & $13(30)$ & $5(45.5)$ \\
\hline CT findings, n (\%) & $33(64.7)$ & $27(70)$ & $6(54.5)$ \\
\hline Unilateral pneumonia & $16(31.4)$ & $13(32.5)$ & $3(27.3)$ \\
\hline Bilateral pneumonia &
\end{tabular}

Table 1. Clinical characteristics of enrolled patients of COVID19 pneumonia.

nucleic acid detection kit to confirm COVID-19 infection. The computed radiography imaging system used by the radiologist was VisionPACS (Intechhosun, Being, China).

Comparison between the model and radiologist in prospective data. The CT scan images of the prospective patients as above were collected and imported into the model for prediction. The model's performance and cost of time were compared with that of the expert radiologist. Inconsistent results between the expert and model were reviewed by three radiologists, including the expert and other two radiologists, senior staff members of the Radiology Department of Renmin Hospital of Wuhan University, with clinical experience about 10 years, and independently diagnosed about 150 viral pneumonia.

Evaluating the efficiency of radiologist with the assistance of Al. To evaluate the performance and cost of time of radiologist against $2019-\mathrm{CoV}$ pneumonia with the assistance of our model, the prediction results of the model (whether a patient has viral pneumonia, and labels marking lesions) were copied to the working computer in the designated CT rooms. After 10 days of wash out period (in Feb 16, 2020), the same expert radiologist was required to re-read all CT images of 27 prospective patients using the working computer where results of the model could be viewed, and determine if each patient has viral pneumonia. The research assistant used a stopwatch to record the expert's reading time again. Hospitalized viral pneumonia cases judged by radiologists were all diagnosed using COVID-19 nucleic acid detection kit to confirm COVID-19 infection. The computed radiography imaging system used by the radiologist was VisionPACS (Intechhosun, Being, China).

Statistical analysis. A two-tailed paired Student's t test with a significance level of 0.05 was used to compare differences in the cost time of the model and radiologist.

\section{Results}

Patients. The baseline characteristics and CT findings of 51 patients of $2019-\mathrm{CoV}$ pneumonia and 55 control patients in retrospective dataset were shown in Tables 1 and 2, respectively. Baseline characteristics were comparable between training and testing datasets. The 31 control patients in retrospective testing dataset include 2 lung cancer, 4 tuberculosis, 2 bronchiectasis, 2 nonviral pneumonia, 1 lung bullae and 20 with no obvious finding in CT scan.

The performance of the model on retrospective dataset. Among $4382 \mathrm{CT}$ images from 11 patients of COVID-19 pneumonia and 9369 images from 31 control patients, the model correctly diagnosed the patients with a per-patient sensitivity of $100 \%$, specificity of $93.55 \%$, accuracy of $95.24 \%$, PPV of $84.62 \%$, and NPV of $100 \%$. A per-image sensitivity of $94.34 \%$, specificity of $99.16 \%$, accuracy of $98.85 \%$, PPV of $88.37 \%$, and NPV of 99.61\%. Representative images predicted by the model were shown in Fig. 5.

The performance of the model in consecutive prospective patients. Twenty-seven patients were enrolled in the prospective dataset in Renmin Hospital of Wuhan University. Sixteen (59.26\%) patients were diagnosed as viral pneumonia by the expert radiologist, and the other eleven patients were not. Two other radiologists reviewed the CT imaging, approved the expert's results, and summarized that the CT characteristics of 


\begin{tabular}{|c|c|c|c|}
\hline & All patients $(n=55)$ & Training set $(n=24)$ & Testing set $(n=31)$ \\
\hline Age, years, median (IQR) & $48(34.5,55)$ & $50.5(38.25,55.25)$ & $47(34.5,54.5)$ \\
\hline \multicolumn{4}{|l|}{ Sex, n (\%) } \\
\hline Men & $31(56.36)$ & $12(50)$ & $19(61.29)$ \\
\hline Women & $24(43.64)$ & $12(50)$ & $12(38.71)$ \\
\hline \multicolumn{4}{|l|}{ CT examination indications, $\mathrm{n}(\%)$} \\
\hline Viral pneumonia to be discharged & $7(12.73)$ & $0(0)$ & $1(3.23)$ \\
\hline Pulmonary bullae to be discharged & $2(3.64)$ & $2(8.33)$ & $0(0)$ \\
\hline Tuberculosis & $1(1.82)$ & $1(4.17)$ & $0(0)$ \\
\hline Lower respiratory infection & $3(5.45)$ & $1(4.17)$ & $2(6.45)$ \\
\hline Metastatic lung tumor to be discharged & $7(12.7)$ & $5(20.83)$ & $2(6.45)$ \\
\hline Routine examination before admission & $41(74.55)$ & $15(62.5)$ & $26(83.87)$ \\
\hline \multicolumn{4}{|l|}{ CT findings, $\mathrm{n}(\%)$} \\
\hline No obvious abnormality & $44(80)$ & 24 & $20(64.52)$ \\
\hline Tuberculous lesion & $4(7.27)$ & - & $4(12.90)$ \\
\hline Suspected neoplastic lesions & $2(3.64)$ & - & $2(6.45)$ \\
\hline Inflammatory lesions (non-viral) & $2(3.64)$ & - & $2(6.45)$ \\
\hline Bronchiectasia & $2(3.64)$ & - & $2(6.45)$ \\
\hline Bullae of lung & $1(1.82)$ & - & $1(3.23)$ \\
\hline
\end{tabular}

Table 2. Clinical characteristics of enrolled control patients.

A

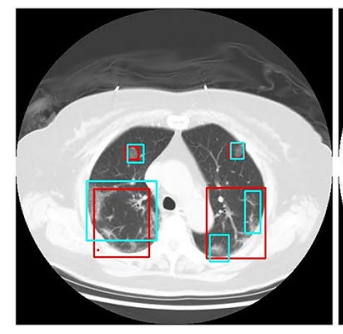

B

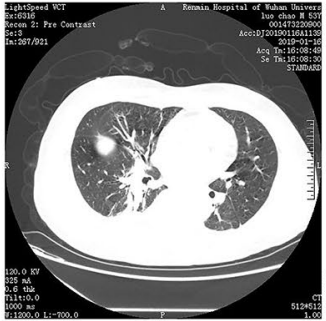

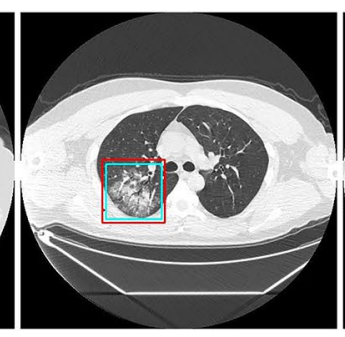

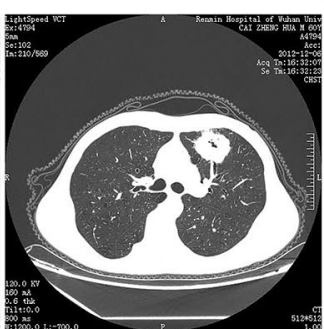

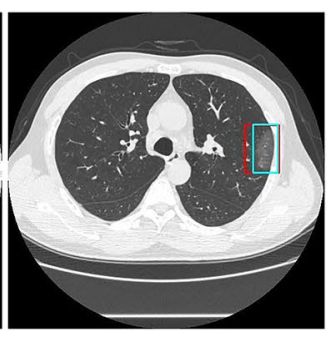

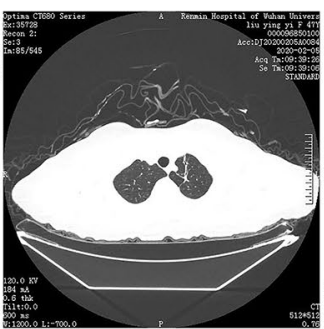

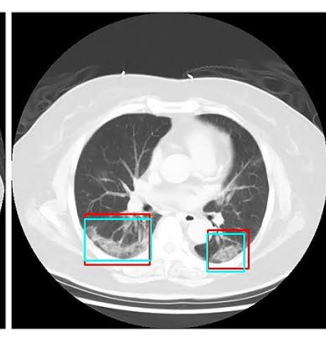
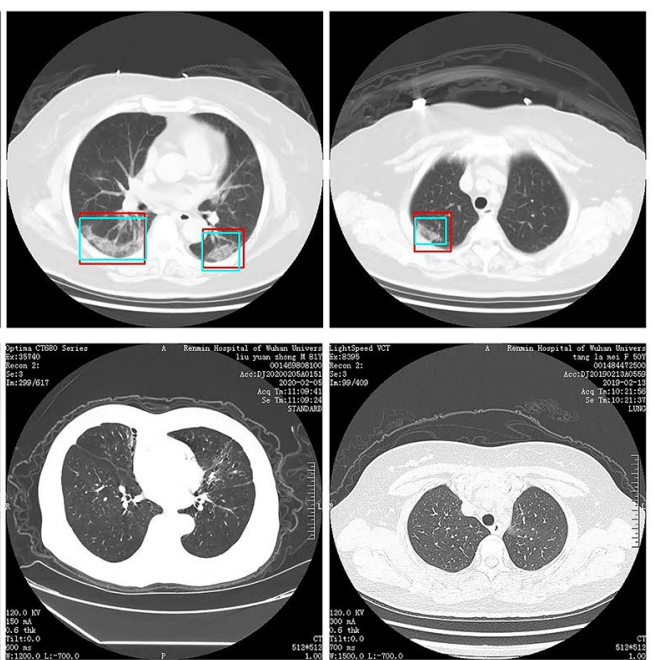

Figure 5. Representative images of the model's predictions. (A) Computed tomography (CT) images of COVID19 pneumonia. The predictions between the artificial intelligence model and radiologists were consistent. Green boxes, labels from radiologists; red boxes, labels from the model. (B) CT images of the control. The first image is an ordinary bacterial pneumonia, showing a consolidation of the right lower lobe. The second image has a tumorous lesion in the lung, showing a mass in the left upper lobe, with spiculation sign seen at the edges, and showing leaf-like growth with vacuoles inside. The third image is a secondary pulmonary tuberculosis, showing a left apical fibrous cord. The fourth image is a bronchiectasis complicated with infection, showing bronchodilation and expansion, cystic changes, and surrounding patches of infection. The fifth image shows normal lungs.

the 11 patients not diagnosed by the expert include 5 ground glass nodules, 3 diminutive nodules, 2 normal and 1 fibrosclerosis.

The model successfully detected all the 16 patients of viral pneumonia diagnosed by the expert. Among the other 11 patients, 2 were also detected by the model. The predictions in one case was fibrosclerosis lesion, and the other one was normal stomach bubble. Using results of the radiologists as the gold standard, the model achieved a per-patient sensitivity of $100 \%$, accuracy of $92.59 \%$, specificity of $81.82 \%$, PPV of $88.89 \%$ and NPV of $100 \%$ in the 27 prospective patients. Among the 16 patients diagnosed as viral pneumonia by radiologists, 8 admitted patients were confirmed as COVID-19 infection, and the others were outpatients that difficult to follow nucleic acid results. The average prediction time for model was 41.34 s per patient (IQR 39.76-44.48). The performance of the model on detecting COVID-19 pneumonia was shown in Table 3. 


\begin{tabular}{|c|c|c|c|c|c|}
\hline & Sensitivity (\%) & Specificity (\%) & Accuracy (\%) & PPV (\%) & NPV (\%) \\
\hline \multicolumn{6}{|l|}{ Internal } \\
\hline \multicolumn{6}{|l|}{ Retrospective testing } \\
\hline Per patient & 100 & 93.55 & 95.24 & 84.62 & 100 \\
\hline Per image & 94.34 & 99.16 & 98.85 & 88.37 & 99.61 \\
\hline Prospective testing (per patient) & 100 & 81.82 & 92.59 & 88.89 & 100 \\
\hline \multicolumn{6}{|l|}{ External } \\
\hline Retrospective testing (per patient) & 98 & 94 & 96 & 94.23 & 97.92 \\
\hline
\end{tabular}

Table 3. The performance of the deep learning model on both retrospective and prospective dataset. $P P V$ positive prediction value; NPV negative prediction value.

Figure 6. Main interface of the open-access artificial intelligence platform which provides fast and sensitive assistance for detecting COVID19 pneumonia.

The performance of the model on external dataset. To estimate the robustness of the system, an external test was conducted using a dataset containing 100 patients from Qianjiang Central Hospital, China. Among them, 50 patients were COVID-19 patients, with a median age of 50 [IQR $(48,58.75)]$ and 32\% $(16 / 50)$ female; the other 50 were normal control patients, with a median age of 40 [IQR $(38.5,51)]$ and $44 \%(22 / 50)$ female. The system achieved an accuracy of $96 \%$, a sensitivity of $98 \%$, a specificity of $94 \%$, a PPV of $94.23 \%$ and an NPV of $97.92 \%$ in external dataset.

Comparison between the efficiency of radiologist with or without the assistance of Al. In the first time the expert radiologist read CT scan images of the 27 prospective patients, the average reading time for him to determine whether each patient has viral pneumonia was $116.12 \mathrm{~s}$ per case (IQR 85.69-118.17). After 10 days of wash out period, the same expert radiologist re-read the CT images of the 27 prospective patients with the assistance of the AI model. The results for determining whether each patient has viral pneumonia were not changed, while the average reading time of the expert was greatly decreased by $65 \%$. This indicates that the efficiency of radiologist could be greatly improved with the assistance of AI.

A website has been made available to provide free access to the present model (https://121.40.75.149/znyxncov/index) (Fig. 6). CT scan images could be uploaded by both clinicians and researches as a second opinion consulting service, especially in other provinces or countries unfamiliar with the radiologic characteristics of COVID-19. Cases of COVID-19 pneumonia were also been made available on the open-access website, which might be a useful resource for radiologists and researchers for fighting COVID-19 pneumonia. Furthermore, the module and source code developed in this work were shared for global researches in https://github.com/ endo-angel/ct-angel. 

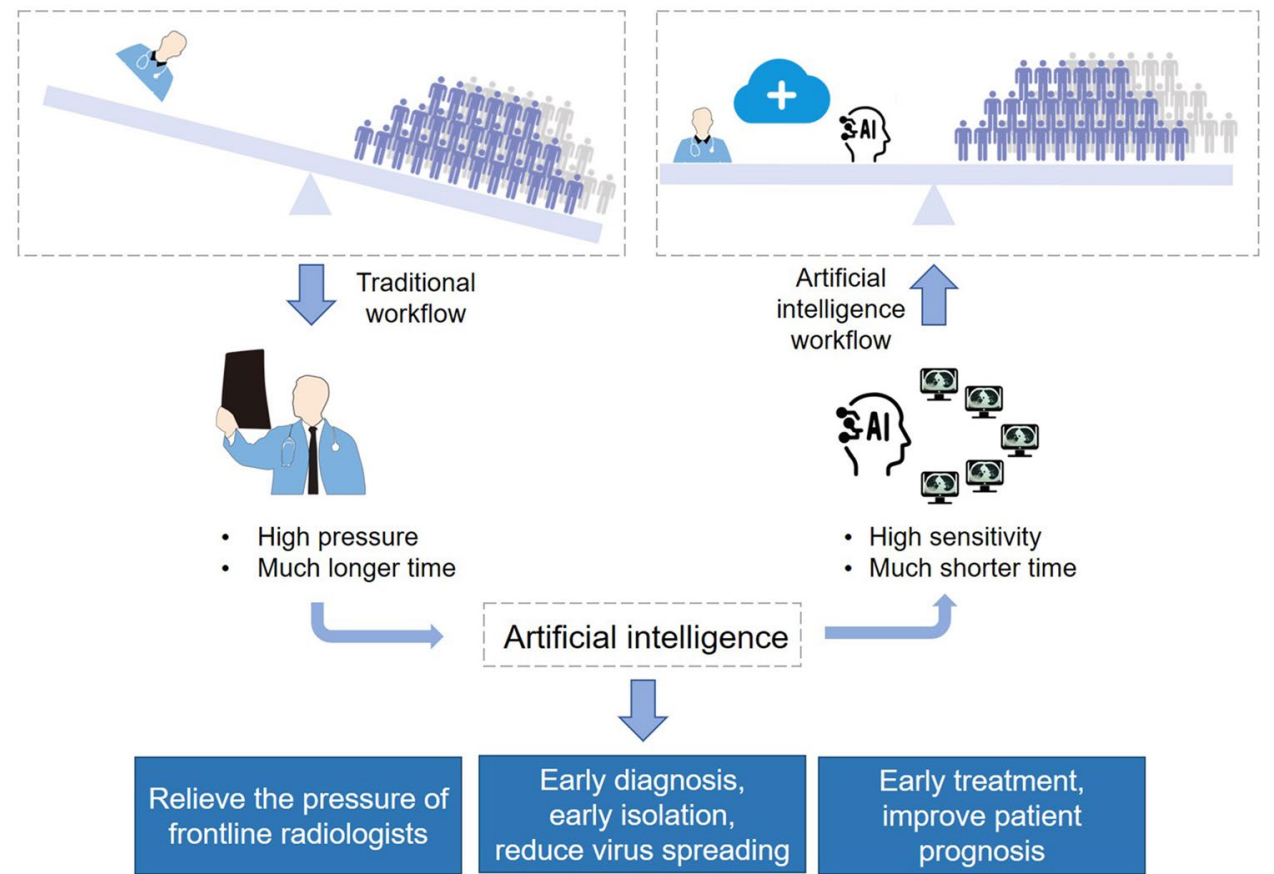

- High sensitivity

- Much shorter time

Figure 7. Abstract diagram. Computed tomography (CT) is the most efficient modality for screening and clinically diagnosing COVID-19 pneumonia. However, compared to the needs of the patients, the number of radiologists is quite small. After enrolling artificial intelligence in identifying COVID-19 pneumonia in CT images, the efficiency of diagnosis is greatly improved. The artificial intelligence holds great potential to relieve the pressure of frontline radiologists, accelerates the diagnosis, isolation and treatment of COVID19 patients, and therefore contribute to the control of the epidemic.

\section{Discussion}

As of Feb 14, 2020, the national health commission had reported 66,492 confirmed cases, 1,523 deaths and 8,969 suspected cases $^{23}$. In the face of such large number of patients and high contagiosity of the novel coronavirus (with an estimated reproduction number R0 of $2.2 \sim 6.47$ ), timely diagnosis and isolation are the keys to prevent further spread of the virus ${ }^{24-28}$. CT scan is the most efficient modality for screening and clinically diagnosing COVID-19 pneumonia ${ }^{5,7}$. However, compared to the needs of the patients, the number of radiologists is quite small, especially in Hubei province, China, which could greatly delay the diagnosis and isolation of patients, affect patient's treatment and prognosis, and ultimately, affect the overall control of COVID-19 epidemic.

Deep learning, a technology has shown great performance on extracting tiny features in radiology data, may hold the promise to alleviate this problem ${ }^{11}$. Recently, Ardila D, et al. achieved end-to-end lung cancer screening on low-dose chest CT with an AUC of $94.4 \%{ }^{29}$. Chae KJ, et al. successfully used the convolutional neural network to classify small $(\leq 2 \mathrm{~cm})$ pulmonary nodules on CT scan images ${ }^{30}$. However, there was rare research being conducted to detect viral pneumonia ${ }^{11,29,30}$. Most previous studies detected pneumonia on X-ray using deep learning while not focused on viral pneumonia. Furthermore, CT is more sensitive and commonly used than X-ray for identifying COVID-19. In our previous work, we succeeded in recruiting deep learning in minor lesion detection and real-time assistance to doctors in gastrointestinal endoscopy ${ }^{12-16}$. Here, we enrolled this technique in identification of COVID-19 pneumonia in CT images. Results from both retrospective and prospective patients showed that the model was comparable to the level of expert radiologist, and hold great potential to reduce diagnosing time. (Fig. 7).

Early diagnosis and early isolation of suspected patients are the most important ways to prevent the spread of epidemic ${ }^{19}$. Due to the sudden outbreak of COVID19, the radiology department is overloaded and patients have to wait for long times for chest CT scan, which largely increase the risk of cross-infection. In recent days, radiologists' daily workload is huge in Hubei province, and a CT scan report has to be awaited several hours to achieve. Based on the number of suspected patients and close contacts in being, radiologists in the hardest hit, Hubei province, China, may not be enough to resist the rapid spread of the virus, which holds high estimated $\mathrm{R} 0$ of $2.2 \sim 6.47^{25-28}$. It could be inferred that before radiologists fulfilling the demands of existing patients, newly infected cases would appear, and the overall burden of radiologists is more overwhelming like a growing snowball. Relieving the pressure of radiologists is essential for the control of virus spreading. In the present study, our model achieved a comparable performance but with much shorter time compared with expert radiologists. It holds great potential to relieve the pressure of radiologists in clinical practice, and contribute to the control of the epidemic.

Timely diagnosis and early treatment of infected patients is important for patients' prognosis ${ }^{31}$. The fatality rate of COVID19 patients in Hubei province is significantly higher than that of other regions, which probably 
due to delayed treatment and shortage of medical resources ${ }^{8,32}$. Accelerating diagnosis efficiency is significant for improving patient outcomes. In the present study, our model helped expert radiologists achieve the same work with much shorter time, which greatly accelerats the efficiency of diagnosis in clinical practice, and may contribute to the improvement of patient outcome.

In addition to relieving radiologists' pressure and accelerating diagnosis efficiency, artificial intelligence also holds the potential to reduce miss diagnosis of COVID-19 patients. The lung infection foci are sometimes mild in the early stage of the COVID-19 infection ${ }^{5}$, and requires careful observation under 0.625 mm layer scanning. Radiologists vary in skills, and could be affected by subjective status and outside pressure. One miss diagnosis could lead to multiple spread. The model is highly sensitive and stable, and would never be affected by work burden and work time. As a preliminary screening tool, it might help radiologists improve the sensitivity and reduce miss diagnosis.

Notably, the sensitivity per patient is better while the other performance per patient is worse than the performance per image. Each patient has a large number of CT images (about 500), most of which were negative images without lesions. The specificity is equal to the true negative divided by all the negatives. The denominator increases hundreds of times when calculating specificity by image, while the numerator (false positive) does not increase so much, therefore, the specificity per image is higher than that of per patient. The same principles could be applied to accuracy and PPV. For sensitivity, a few images having suspicious lesions may be missed in COVID19 patients (sensitivity per image), while the probability that all images having suspicious lesions in a patient would be much lower (sensitivity per patient).

On the basis of the accuracy and efficiency of the model in detecting COVID-19 pneumonia, a cloud-based open-access artificial intelligence platform was constructed to provide assistance for detecting COVID-19 pneumonia worldwide. CT scan images could be uploaded freely by both clinicians and researches as an assistant tool, especially in other provinces or countries unfamiliar with the radiologic characteristics of COVID-19. This free open-access website can read images in batches, provide high-level auxiliary diagnostic services for different hospitals in free, and expand the boundaries of regions and manpower. Cases of COVID-19 pneumonia were also been made available on the open-access website, which might be a useful resource for radiologists and researchers for fighting COVID-19 pneumonia.

In summary, the deep learning-based model achieved a comparable performance with expert radiologist using much shorter time. It holds great potential to improve the efficiency of diagnosis, relieve the pressure of frontline radiologists, accelerates the diagnosis, isolation and treatment of COVID19 patients, and therefore contribute to the control of the epidemic.

Received: 24 March 2020; Accepted: 3 August 2020

Published online: 05 November 2020

\section{References}

1. Hui, D. S. et al. The continuing COVID-19 epidemic threat of novel coronaviruses to global health-The latest 2019 novel coronavirus outbreak in Wuhan, China. Int. J. Infect. Dis. 91, 264-266 (2020).

2. Holshue, M. L. et al. First case of 2019 novel coronavirus in the United States. N. Engl. J. Med. 382(10), 929-936 (2020).

3. Rothe, C. et al. Transmission of COVID-19 Infection from an asymptomatic contact in Germany. N. Engl. J. Med. 382(10), 970-971 (2020).

4. Huang, C. et al. Clinical features of patients infected with 2019 novel coronavirus in Wuhan, China. Lancet 395(10223), 497-506 (2020).

5. Guan, H. et al. Clinical and thin-section CT features of patients with the COVID-19 in Wuhan (in Chinese). Radiol. Pract. 35, 125-130 (2020).

6. Chen, N. et al. Epidemiological and clinical characteristics of 99 cases of 2019 novel coronavirus pneumonia in Wuhan, China: a descriptive study. Lancet 395(10223), 507-513 (2020).

7. National Health and Health Commission of China. The interpretation of Diagnosis and Treatment Protocol for COVID-19 (Trial Version 5) in Chinese. https://www.nhc.gov.cn/yzygj/s7652m/202002/e84bd30142ab4d8982326326e4db22ea.shtml (2020).

8. National Health and Health Commission of China. Feb 13: Daily briefing on novel coronavirus cases in China. https://en.nhc.gov. $\mathrm{cn} / 2020-02 / 13 /$ c_76512.htm (2020).

9. Health Commission of Hubei Province. Covid-19 outbreak in hubei province on 15 February 2020. https://wjw.hubei.gov.cn/fbjd/ dtyw/202002/t20200216_2038684.shtml (2020).

10. China Ministry of Health. China Health Statistical Yearbook 2018[in Chinese]. 34-42 (Beijing: Peking Union Medical College Press, 2018).

11. Liu, Y., Liu, G. \& Zhang, Q. Deep learning and medical diagnosis. Lancet 394(10210), 1709-1710 (2019).

12. Gong, D. et al. Detection of colorectal adenomas with a real-time computer-aided system (ENDOANGEL): a randomised controlled study. Lancet Gastroenterol. Hepatol. 5(4), 352-361 (2020).

13. Wu, L. et al. Randomised controlled trial of WISENSE, a real-time quality improving system for monitoring blind spots during esophagogastroduodenoscopy. Gut 68(12), 2161-2169 (2019).

14. Chen, D. et al. Comparing blind spots of unsedated ultrafine, sedated, and unsedated conventional gastroscopy with and without artificial intelligence: a prospective, single-blind, 3-parallel-group, randomized, single-center trial. Gastrointest. Endosc. 91(2), 332-339 (2020).

15. Zhou, J. et al. A novel artificial intelligence system for the assessment of bowel preparation (with video). Gastrointest. Endosc. 91(2), 428-435 (2020).

16. Wu, L. et al. A deep neural network improves endoscopic detection of early gastric cancer without blind spots. Endoscopy 51(06), 522-531 (2019).

17. The Central People's Government of the People's Republic of China. The Law of the People's Republic of China on Infectious Disease Prevention and Control. https://www.gov.cn/banshi/2005-08/01/content_19023.htm (2005).

18. Centers for Disease Control and Prevention. Information for Laboratories about Coronavirus (COVID-19). https://www.cdc.gov/ coronavirus/2019-ncov/lab/index.html (2020).

19. Zhou, Z., Siddiquee, M. M. R., Tajbakhsh, N. \& Liang, J. Unet++: a nested u-net architecture for medical image segmentation. Preprint at. https://arxiv.org/abs/1807.10165 (2018). 
20. Chen, L., Papandreou, G., Schroff, F. \& Adam, H. Rethinking atrous convolution for semantic image segmentation. Preprint at. https://arxiv.org/abs/1706.05587 (2017).

21. He, K., Zhang, X., Ren, S. \& Sun, J. Deep residual learning for image recognition. IEEE conference on computer vision and pattern recognition. $770-778,(2016)$.

22. Deng, J., Dong, W., Socher, R., Li, L. \& Li, F. ImageNet: a large-scale hierarchical image database. IEEE Conference on Computer Vision and Pattern Recognition.248-255 (2009).

23. The National Health and Health Commission of China. Feb 7: Daily briefing on novel coronavirus cases in China. https://en.nhc. gov.cn/2020-02/07/c_76323.htm (2020).

24. Cheng, V. C., Wong, S., To, K. K., Ho, P. L. \& Yuen, K. Preparedness and proactive infection control measures against the emerging Wuhan coronavirus pneumonia in China. J. Hosp. Infect. 104(3), 254-255 (2020).

25. Tang, B. et al. Estimation of the transmission risk of the $2019-\mathrm{nCoV}$ and its implication for public health interventions. J. Clin. Med. 9(2), $462(2020)$.

26. Riou, J. \& Althaus, C. L. Pattern of early human-to-human transmission of Wuhan 2019 novel coronavirus (2019-nCoV), December 2019 to January 2020. Euro Surveill. 25(4), 2000058 (2020).

27. Prasse, B., Achterberg, M. A., Ma, L. \& Van Mieghem, P. Network-based prediction of the 2019-nCoV epidemic outbreak in the Chinese Province Hubei. Preprint at. https://arxiv.org/abs/2002.04482 (2020).

28. Boldog, P., Tekeli, T., Vizi, Z., Denes, A., Bartha, F. A. \& Rost, G. Risk assessment of novel coronavirus 2019-nCoV outbreaks outside China. Preprint at. https://www.medrxiv.org/ (2020).

29. Ardila, D. et al. End-to-end lung cancer screening with three-dimensional deep learning on low-dose chest computed tomography. Nat. Med. 25(6), 954-961 (2020).

30. Chae, K. J. et al. Deep learning for the classification of small $(\leq 2 \mathrm{~cm})$ pulmonary nodules on CT imaging: a preliminary study. Acad. Radiol. 27(4), e55-e63 (2020).

31. Chen, Z. et al. Diagnosis and treatment recommendations for pediatric respiratory infection caused by the 2019 novel coronavirus. World J. Pediatr.. https://doi.org/10.1007/s12519-020-00345-5 (2020).

32. Health Commission of Hubei Province. Covid-19 outbreak in hubei province on 14 February 2020. https://wjw.hubei.gov.cn/fbjd/ dtyw/202002/t20200215_2028355.shtml (2020).

\section{Acknowledgements}

This work was partly supported by the Grant from Project of Hubei Provincial Clinical Research Center for Digestive Disease Minimally Invasive Incision (Grant No. 2018BCC337), Hubei Province Major Science and Technology Innovation Project (Grant No. 2018-916-000-008), the National Natural Science Foundation of China [Grant Nos. 81672387 to Yu Honggang].

\section{Author contributions}

$\mathrm{YH}, \mathrm{YL}$ and CF conceived and supervised the overall study. WL and GD contributed to writing of the manuscript. $\mathrm{YH}, \mathrm{YL}, \mathrm{HS}$ and WY contributed to critical revision of the report. CJ, ZL, ZJ, GD, WH, DZ, XY, ZY, CX, ZM, CQ, HS, YM and YX contributed to collecting and analyzing the data of patients. CJ, ZL and ZY contributed to label CT images. HS, HX, ZB, ZK and WY developed the system. All authors reviewed and approved the final version of the manuscript. The funder of the study had no role in study design, data collection, data analysis, data interpretation, or writing of the report. The corresponding author had full access to all the data in the study and had final responsibility for the decision to submit for publication.

\section{Competing interests}

The authors declare no competing interests.

\section{Additional information}

Supplementary information is available for this paper at https://doi.org/10.1038/s41598-020-76282-0.

Correspondence and requests for materials should be addressed to L.Y., F.C. or H.Y.

Reprints and permissions information is available at www.nature.com/reprints.

Publisher's note Springer Nature remains neutral with regard to jurisdictional claims in published maps and institutional affiliations.

(c) (i) Open Access This article is licensed under a Creative Commons Attribution 4.0 International License, which permits use, sharing, adaptation, distribution and reproduction in any medium or format, as long as you give appropriate credit to the original author(s) and the source, provide a link to the Creative Commons licence, and indicate if changes were made. The images or other third party material in this article are included in the article's Creative Commons licence, unless indicated otherwise in a credit line to the material. If material is not included in the article's Creative Commons licence and your intended use is not permitted by statutory regulation or exceeds the permitted use, you will need to obtain permission directly from the copyright holder. To view a copy of this licence, visit http://creativecommons.org/licenses/by/4.0/.

(c) The Author(s) 2020 